\title{
16-kDa Prolactin Reduces Angiogenesis, but Not Growth of Human Breast Cancer Tumors In Vivo
}

\author{
Jessica M. Faupel-Badger • Erika Ginsburg • \\ Jodie M. Fleming • Leah Susser • Teresa Doucet • \\ Barbara K. Vonderhaar
}

Published online: 11 March 2010

(C) U.S. Government 2010

\begin{abstract}
Prolactin (PRL) is a peptide hormone necessary for normal growth and development of the human breast. In addition, high levels of PRL in plasma correlate with increased risk of breast cancer, especially among postmenopausal women. Several isoforms of PRL exist in human circulation, including a $16-\mathrm{kDa}$ isoform that is an N-terminal fragment of the full-length 23-kDa PRL. 16-kDa PRL has been shown to be antiangiogenic in vitro and in vivo and to reduce formation of tumors from prostate, colon, and melanoma cancer cell lines. Here, we explore the effect of 16-kDa PRL expression in vitro and in vivo using two breast cancer cell line models (MCF-7 and MDA-MB-231) and also the HCT-116 colon cancer cell line. In all three cell lines, 16-kDa PRL expression inhibited cell proliferation in vitro compared to empty vector controls. In vivo results were markedly different between the two types of cell lines. HCT-116 cells expressing 16-kDa PRL exhibited reduced vascularization and tumor formation, consistent with published results. The breast cancer cell lines expressing 16-kDa PRL also exhibited inhibition of angiogenesis in vivo but no reduction in tumor size or formation. These results suggest that the effects of $16-\mathrm{kDa} P R L$ on tumor
\end{abstract}

Jessica M. Faupel-Badger and Erika Ginsburg equally contributed to this work.

J. M. Faupel-Badger · E. Ginsburg · J. M. Fleming $\cdot$ L. Susser •

T. Doucet $\cdot$ B. K. Vonderhaar

Mammary Biology and Tumorigenesis Laboratory,

Center for Cancer Research,

Bethesda, MD 20892-7105, USA

Present Address:

J. M. Faupel-Badger ( $\square)$

Cancer Prevention Fellowship Program, Center for Cancer

Training, National Cancer Institute,

6120 Executive Blvd (EPS), Suite 150E, MSC 7105,

Bethesda, MD 20892-7105, USA

e-mail: badgerje@mail.nih.gov formation may vary across tissue types. The unique sensitivity of breast cancer to PRL as a mitogen and/or additional factors in the mammary gland environment (e.g., local hormone/ mitogen concentration) may play a dominant role in tumor formation in vivo, thus outweighing the antiangiogenesis effects and in vitro reduction in cell proliferation induced by 16-kDa PRL.

Keywords Prolactin - Angiogenesis · Proliferation . Breast cancer - Vasoinhibins

\section{Introduction}

Prolactin (PRL) is responsible for the growth and development in normal human breast and plays a role in breast cancer risk. High PRL levels in serum collected prospectively from postmenopausal women has been associated with increased risk of developing breast cancer (relative risk 1.3, 95\% CI 1.1-1.6) [1]. Positive associations between high PRL levels and risk factors for breast cancer, including nulliparity, oral contraceptive use, and family history of breast cancer, have also been reported [2]. In vitro, PRL enhances proliferation, migration, and survival of human breast cancer cells [2]. PRL exerts these effects through binding to the PRL receptor (PRLR) on the surface of normal human breast epithelial and cancer cells, thereby initiating the Jak2/Stat5, PI3K, and mitogen-activated protein kinase (MAPK) signaling pathways [2].

PRL functions in both an endocrine manner, where it is secreted by the pituitary and acts on distant tissues such as the mammary gland, and in an autocrine/paracrine manner whereby normal breast epithelial and breast tumor cells synthesize and secrete their own PRL [3]. In addition, multiple PRL isoforms have been detected in circulation. 
The $23-\mathrm{kDa}$ isoform is the most common isoform in circulation [4]. The vast majority of in vitro and in vivo studies of PRL, particularly in the mammary gland, have focused on the $23-\mathrm{kDa}$ isoform; therefore, most of the actions of PRL have been attributed to this isoform. In addition to its functions in proliferation, apoptosis, migration, and differentiation, 23-kDa PRL has been shown to be proangiogenic $[5,6]$. Interestingly, another isoform, $16-\mathrm{kDa}$ PRL, functions in direct opposition to 23-kDa PRL where it has been shown to be antiangiogenic [7].

$16-\mathrm{kDa}$ PRL is a posttranslational cleavage product of 23-kDa PRL $[8,9]$ and can be detected in serum and other human samples [4, 9-13]. Although the role of 16-kDa PRL in normal and pathologic conditions is just beginning to be explored, higher levels of 16-kDa PRL have been positively associated with preeclampsia [12] and postpartum cardiomyopathy [13], two detrimental pregnancy-associated conditions that involve alterations in angiogenesis. Conversely, it has been suggested that antiangiogenic effects of $16-\mathrm{kDa}$ PRL expression may be beneficial in cases of retinopathy of prematurity [11], which is a condition marked by excess angiogenesis.

16-kDa PRL can be generated by cathepsin D cleavage following amino acid (aa) 150, and this specific cleavage product has been detected in pituitary adenoma samples [9]. Other sites of cathepsin D cleavage, at least in vitro, follow aa 132 or 147 and produce PRL isoforms of approximately $16 \mathrm{kDa}$ [9]. All three of these cleavage products demonstrate antiangiogenic activity by inhibiting proliferation and migration of endothelial cells in vitro and vascularization in the chick allantoic membrane assay [9]. Collectively, the term vasoinhibins is used to refer to this family of N-terminal PRL cleavage products with antiangiogenic activity [7].

Studies of $16-\mathrm{kDa}$ PRL in experimental systems have largely focused on endothelial cells; therefore, little is known about the functions of 16-kDa PRL in normal epithelial and tumor cells. Three independent studies, one using a colon cancer cell line [14], one using prostate cancer cell lines [15], and one using a melanoma cell line [16], have attempted to address the functions of $16-\mathrm{kDa}$ PRL. Of note, the recombinant $16-\mathrm{kDa}$ PRL isoforms used in the colon, prostate, and melanoma studies were established before the sites of cleavage with cathepsin D were determined [9]. Specifically, the HCT-116 colon cancer cells were stably transfected with a $16-\mathrm{kDa}$ PRL construct truncated at aa 139 [14] and the prostate and melanoma cell lines were expressing $16 \mathrm{kDa}-$ PRL truncated at aa $123[15,16]$. Although truncation at aa 123 and 139 was not observed with cathepsin D cleavage or in vivo, these recombinant PRL proteins of approximately $16 \mathrm{kDa}$ also display antiangiogenic properties with respect to endothelial cell proliferation and in the chick allantoic membrane assay $[15,17]$.
When HCT-116 colon cancer cells expressed 16-kDa PRL (aa 1-139), the in vitro growth properties of the cells were not affected [14]. Injection of these cells into Rag1-/- mice resulted in no difference in the number of tumors formed, compared to empty vector control cells. However, the authors found that the HCT-116-16-kDa PRL tumors were smaller than the control tumors and appeared to have less microvessel density. For the prostate cancer study, DU145 and PC-3 cells were infected with an adenovirus construct containing $16-\mathrm{kDa}$ PRL truncated at aa 123 [15]. The 16-kDa PRL expression again did not alter the in vitro growth properties of either prostate cancer cell line; however, subcutaneous injection of these cells into immunocompromised mice resulted in no tumor formation, while emptyvector-transfected cells formed tumors. Injection of the adenovirus-16-kDa PRL construct into mice with established tumors resulted in regression of the tumors. Similarly, introduction of 16-kDa PRL into the subcutaneous B16/ F10 mouse melanoma model via adenovirus-mediated gene transfer significantly delayed tumor development [16]. Additionally, this study showed a $50 \%$ reduction in lung metastasis size and number after 16-kDa PRL exposure.

The results from these prior studies, together with the numerous studies documenting the antiangiogenic effects of 16-kDa PRL, led us to hypothesize that 16-kDa PRL may also have antitumorigenic effects in breast cancer cells. To test the effects of 16-kDa PRL in a breast cancer model, full-length PRL was truncated at aa 150 to produce $16-\mathrm{kDa}$ PRL as detected in pituitary adenoma samples. A 16-kDa PRL was overexpressed in estrogen receptor (ER)-positive and PRLRpositive MCF-7 and ER-negative low-PRLR MDA-MB-231 breast cancer cell lines. We show that the 16-kDa PRL isoform is functionally active in a bioassay using cells extremely sensitive to PRL as measured by proliferation in response to addition of this lactogen. The in vitro effects of $16-\mathrm{kDa}$ PRL were similar in both breast cancer cell lines even though the cell lines greatly differed in ER and PRLR expression. The HCT-116 human colon carcinoma cell line was used as a positive control since previous studies demonstrated that $16-\mathrm{kDa}$ PRL resulted in smaller tumors in vivo and did not decrease cell proliferation in vitro [14]. The results presented herein demonstrate that $16-\mathrm{kDa}$ PRL expression, while inhibiting cell proliferation in vitro and angiogenesis in vivo, has no effect on tumor formation in vivo using the MDAMB-231 or MCF-7 breast cancer cell lines.

\section{Materials and Methods}

Cells

MDA-MB-231, MCF-7, and HCT-116 cells were obtained from the American Type Culture Collection (ATCC; 
Manassas, VA, USA). MDA-MB-231 cells were routinely grown in RPMI1640 medium in the presence of 5\% fetal bovine serum (FBS; Invitrogen, Gaithersburg, MD, USA); MCF-7 were passaged in DMEM with 5\% FBS, and HCT116 cells were maintained in McCoys 5a medium with $5 \%$ FBS.

Generation of the 16-kDa PRL Construct and Stable Cell Lines

The 16-kDa PRL construct was created using the Stratagene (La Jolla, CA, USA) QuikChange ${ }^{\circledR}$ XL Site-Directed Mutagenesis Kit and primers $5^{\prime}$ CCCTGTCTGGTAGG GACTTCCATCCCTG and 5'CAGGGATGGAAGTCCC TACCAGACAGGG to introduce a stop codon at amino acid 151 into the human PRL cDNA (ATCC) expressed in the pEF6-His plasmid (Invitrogen). MDA-MB-231, MCF-7, and HCT-116 cells were stably transfected with either the $16-\mathrm{kDa}$ PRL construct or empty vector (EV) using FuGENE 6 (Roche, Indianapolis, IN, USA). The cells were selected for 4 weeks with $2 \mu \mathrm{g} / \mathrm{ml}$ blasticidin (Invitrogen). Transfected cells were clonally selected; four to six separate clones from each cell line were tested, and the cell line with the highest expression of $16-\mathrm{kDa}$ PRL as determined by reverse transcription-polymerase chain reaction (RT-PCR) was used for all experiments. Experiments were then performed on the stably selected populations that were continuously maintained in blasticidin-containing media.

\section{RT-PCR}

Total RNA was extracted from the stable cell lines using Trizol (Invitrogen), and $2 \mu \mathrm{g}$ of RNA was used for reverse transcriptase (RT) reactions. The RT and PCR reactions were performed using the conditions and primers previously published $[18,19]$. The expected PCR product sizes are $612 \mathrm{bp}$ for PRL and $983 \mathrm{bp}$ for GADPH.

\section{In Vitro Growth Assay}

Plated in triplicate in six-well culture dishes for each cell type were $5 \times 10^{4}$ cells. Cells were plated in media with $5 \%$ FBS; the following day, the media containing FBS were removed and replaced with media containing $5 \%$ charcoal stripped serum (CSS). Cells were counted each day for 3 days after plating using a Beckman Coulter counter. Each growth assay was repeated two (MCF-7 and HCT-116) or three (MDA-MB-231) times in triplicate.

\section{Nb2 Cell Growth Bioassay}

$\mathrm{Nb} 2$ cells, a highly PRL-responsive rat lymphoma cell line (originally obtained from Dr. Peter Gout, British Columbia
Cancer Agency, Vancouver, BC, Canada), were grown as previously described [3]. Concentrations of human $23-\mathrm{kDa}$ PRL (Sigma-Aldrich, St. Louis, MO, USA) were added to create a standard curve. Growth of $\mathrm{Nb} 2$ cells is linear in the range of $1-500 \mathrm{pg} / \mathrm{ml}$ PRL. For the experimental groups, $50 \mu$ of conditioned media was added. After an additional 3 days in culture, cells were collected by centrifugation and counted in a Coulter counter. Cell viability was $>95 \%$. All growth experiments were performed at least two times with triplicate cultures.

\section{Preparation of Conditioned Media}

MDA-MB-231, MCF-7, or HCT-116 cells stably expressing either EV or 16-kDa PRL were plated in T-25 flasks $\left(25 \mathrm{~cm}^{2}\right)$ in media containing FBS and allowed to grow for 2 days or until approximately $80 \%$ confluency. The cells were then grown in serum-free conditions for 1 day. The conditioned medium was collected and frozen for later use.

\section{Tumor Formation in Nude Mice}

Six-week-old female athymic nu/nu mice were purchased from the NCI colony (APA, Frederick, MD, USA). All animals were maintained on a 12-h light/12-h dark schedule with free access to laboratory chow and water. All animal experiments were conducted in accord with the accepted standards of humane animal care and approved by the Animal Care and Use Committee at the National Institutes of Health. For the orthotopic injections, $2 \times 10^{6} \mathrm{MCF}-7$ or MDA-MB-231 cells stably expressing either EV or 16-kDa PRL were suspended in $100 \mu \mathrm{l}$ of sterile phosphatebuffered saline (PBS) and injected into the abdominal mammary gland (ten animals per transfected cell line). Mice injected either orthotopically or subcutaneously with MCF-7 cells also received a subcutaneous estrogen pellet (0.72 mg $\beta$-estradiol, 90-day release, Innovative Research of America, Sarasota, FL, USA). For subcutaneous injections, $2 \times 10^{6}$ MCF-7, MDA-MB-231, or HCT-116 cells stably expressing either EV or $16-\mathrm{kDa}$ PRL cells were suspended in $100 \mu \mathrm{l}$ of sterile PBS and injected in the flank (five animals per transfected cell line for MCF-7 and HCT116 , ten animals per transfected cell line for MDA-MB231). Once palpable, tumor size was measured (length $\times$ width, square centimeter) with calipers twice weekly during weeks 4-8. Experiments were terminated when any animal in the group had a tumor that reached the maximal allowable size as determined by the NIH IACUC. All animals were sacrificed at this time ( 8 weeks postinjection for these studies) so that final tumor measures could be compared between EV and 16-kDa PRL groups. The tumors were then either frozen or formalin-fixed for immunohistochemistry analysis. 


\section{Ki-67 Staining}

Formalin-fixed, paraffin-embedded tumor sections were stained for Ki-67 (Santa Cruz Biotechnology, Santa Cruz, CA, USA) using the Elite Vectastain kit (Vector Laboratories, Burlingame, CA, USA) according to the manufacturer's instructions. Rabbit anti-Ki-67 was incubated on the slides for $1 \mathrm{~h}$ at room temperature followed by antirabbit secondary antibody (GE Healthcare) for $30 \mathrm{~min}$. Staining was visualized using the $\mathrm{DAB}$ reaction; slides were washed, counterstained with hematoxylin, and mounted. Five photos at $40 \mathrm{X}$ from random fields of each tumor section were taken. The percent of cells that were positive (proliferating) was determined for each photo. Photos of the whole tumor were taken at $4 \mathrm{X}$ to confirm that the $40 \mathrm{X}$ photos were representative of the tumor. Two sections from each tumor were stained and counted in separate experiments. All tumors from MCF-7 and MDA-MB-231 expressing either EV or $16-\mathrm{kDa}$ PRL were stained for Ki67 antigen.

\section{The Directed In Vivo Angiogenesis Assay}

The effects of 16-kDa PRL expression in MCF-7, MDAMB-231, and HCT-116 cells on angiogenesis in vivo was evaluated using the directed in vivo angiogenesis assay (DIVAA) kit from Trevigen (Gaithersburg, MD, USA). Placed into each angioreactor were $2 \times 10^{5}$ cells in $20 \mu \mathrm{l}$ basement membrane extract (BME). Positive (5 $\mu$ l fibroblast growth factor (FGF)/vascular endothelial growth factor (VEGF)) and negative (5 $\mu \mathrm{l}$ PBS) control angioreactors (three mice per experiment) were included in each experiment. Angioreactors were implanted subcutaneously into athymic nu/nu mice. Each mouse had two angioreactors implanted, one EV and one $16 \mathrm{kDa}$, subcutaneously in the dorsal region (five mice per experiment). The angioreactors were removed after 9 days and photographed, and the presence of blood vessels was quantified using NIH ImageJ. The DIVAA experiment was repeated twice for each cell type.

\section{Fluorescent Visualization of Vasculature}

Once the MCF-7 tumors reached $0.25 \mathrm{~cm}^{2}$, the mice were injected via tail vein with fluorescein isothiocyanatedextran (FITC-dextran, $2 \times 10^{6} \mathrm{MW}$; Sigma, St. Louis, MO, USA) to permit visualization of functional blood vessels [20]. After $15 \mathrm{~min}$, the animals were euthanized, and tumors were collected and fixed in $4 \%$ paraformaldehyde overnight. The fixed tumors were then placed in OCT; frozen sections were prepared immediately; blood vessels were visualized using the Carl Zeiss LSM510 confocal imaging system (Carl Zeiss Micro Imaging, Thornwood,
NY, USA) at 40X magnification and quantified using NIH ImageJ.

\section{Statistical Analyses}

Student's $t$ tests were used to examine differences in mean tumor weight and size or mean cell counts between EV- and 16-kDa-PRL-expressing cells from the same parent cell line (e.g., MCF-7). Tumor size and weight also were examined using the nonparametric Mann-Whitney $U$ test. The DIVAA and FITC-dextran results were analyzed using both paired $t$ tests and nonparametric Mann-Whitney $U$ tests. Significance was determined at the $p \leq 0.05$ level.

\section{Results}

The ER-positive and PRLR-positive MCF-7 and the ERnegative low-PRLR MDA-MB-231 cell lines were used to assess the effects of $16-\mathrm{kDa}$ PRL expression in breast cancer cell lines and to determine whether the observed effects of 16-kDa PRL expression varied depending upon ER or PRLR expression. Expression of the 16-kDa PRL construct in stably transfected cell lines was confirmed using RT-PCR with primers that would recognize a region of PRL common to both the $16-\mathrm{kDa}$ and the $23-\mathrm{kDa}$ PRL isoforms. The human breast cancer cell line T47D produces endogenous $23 \mathrm{kDa}$ PRL and was used as a positive control for PRL expression (Fig. 1a). The MDA-MD-231 and MCF-7 clones used in this study do not make endogenous PRL, as can be seen in the lanes corresponding to the empty vector/negative controls in Fig. 1a. Therefore, in the 16-kDa PRL transfected cell lines, the band in Fig. 1a for both the MDA-MB-231 and MCF-7 cells is due to expression of the 16-kDa PRL construct. HCT-116 human colon carcinoma cells also do not produce endogenous PRL as seen in the empty vector lane in Fig. 1b. Expression of $16-\mathrm{kDa}$ PRL can be detected in the stable HCT-116 transfectants (Fig. 1b).

$\mathrm{The} \mathrm{Nb} 2$ rat lymphoma cell line is exquisitely sensitive to the mitogenic effects of PRL even at low (i.e., picogram) levels [21]. Therefore, PRL concentrations in serum or conditioned media can be quantified by measuring the increase in cell proliferation induced by the sample in comparison to known PRL standards. To determine if the 16-kDa PRL expression construct was producing a biologically active, secreted PRL isoform, conditioned media were collected from MDA-MB-231, MCF-7, and HCT-116 cells stably expressing either EV or $16-\mathrm{kDa}$ PRL. The concentration of $16-\mathrm{kDa}$ PRL in the conditioned media from each cell type is reported in Fig. 1c. The two breast cancer cell lines secreted similar levels of 16-kDa PRL at approximately $30 \mathrm{pg} / \mathrm{ml}$ per $24 \mathrm{~h}$ per $10^{6}$ cells and the HCT- 


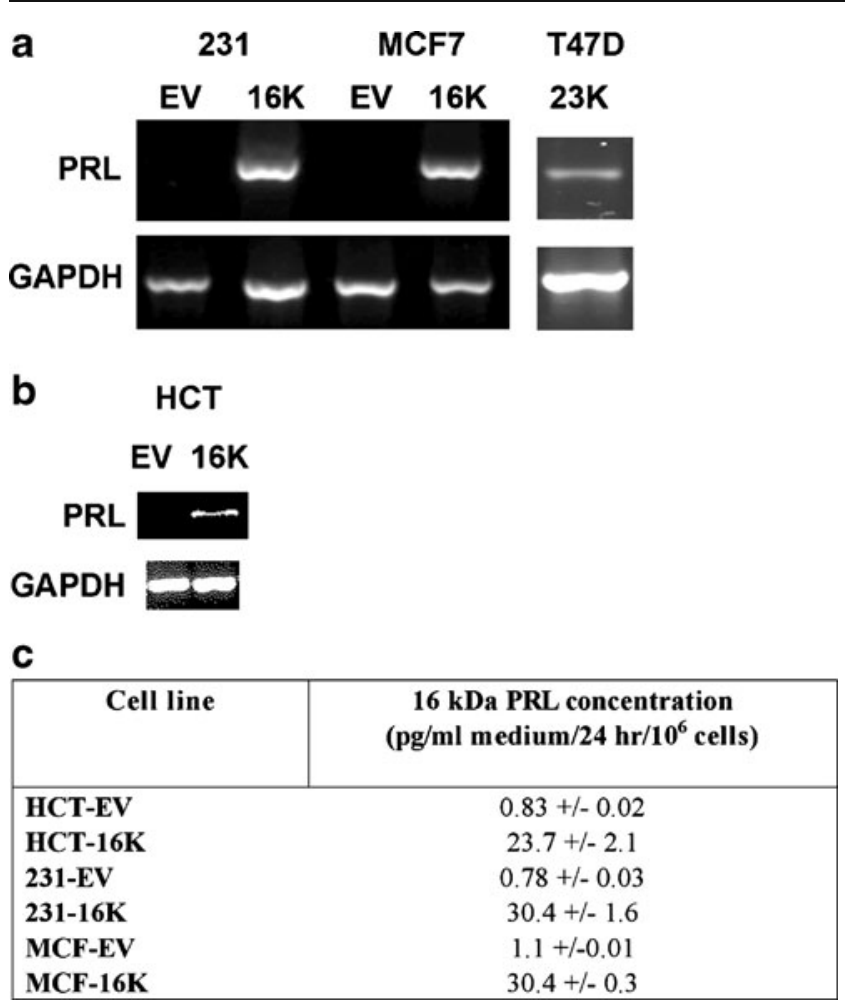

Fig. 1 RT-PCR confirms PRL expression. a MDA-MB-231 (231) and MCF-7 breast cancer cell lines stably transfected with $16-\mathrm{kDa}$ PRL $(16 K)$ express $16-\mathrm{kDa}$ PRL mRNA. T47D cells that produce autocrine $23-\mathrm{kDa}$ PRL were used as a positive control. The top panel depicts a band corresponding to PRL. The bottom panel depicts a band corresponding to GAPDH, a housekeeping gene. b HCT-116 $(H C T)$ colon cell line stably transfected with $16-\mathrm{kDa}$ PRL (16K) express $16-\mathrm{kDa}$ PRL mRNA. The bottom panel corresponds to GAPDH. c A 16-kDa PRL concentration determined by Nb2 bioassay. Conditioned media from MDA-MB-231, MCF-7, and HCT-116 cells transfected with either EV or 16-kDa PRL were collected, and the concentration of $16-\mathrm{kDa}$ PRL in the media was determined using the $\mathrm{Nb} 2$ bioassay. All three 16-kDa PRL transfected cells lines exhibited substantially more activity in the $\mathrm{Nb} 2$ bioassay than the EV controls. $E V$ empty vector control.

116/16-kDa PRL cells secreted slightly less at approximately $24 \mathrm{pg} / \mathrm{ml}$ per $24 \mathrm{~h}$ per $10^{6}$ cells. These values are from direct comparison of the unknowns to the standard curve obtained with known 23-kDa PRL concentrations. It has been previously reported that $16-\mathrm{kDa}$ PRL exhibits $60 \%$ of the mitogenic activity of $23-\mathrm{kDa}$ PRL in the $\mathrm{Nb} 2$ bioassay [22]. Therefore, the actual concentrations of $16-\mathrm{kDa}$ PRL secreted by each cell line may be up to $40 \%$ higher than the values reported in Fig. 1c.

To determine the effects of $16-\mathrm{kDa}$ PRL expression on cell proliferation in human cancer cells, MDA-MB-231, MCF-7, and HCT-116 cells stably expressing either empty vector or $16-\mathrm{kDa}$ PRL were grown on tissue culture plastic in the presence of $5 \%$ CSS. Charcoal stripping of serum removes many of the endogenous hormones and growth factors including lactogens that could interfere with the interpretation of the results from the media. In all three cell lines, cells expressing 16-kDa PRL proliferated at a reduced rate compared to empty vector transfected cells (Fig. 2). Both breast cancer cell lines exhibited statistically significant differences between EV- and 16-kDa-PRL-expressing cells with $p=0.001$ for day 4 comparison of MDA-MB-231 cells and $p=0.009$ and 0.01 for days 3 and 4 comparisons, respectively, of MCF-7 cells. The HCT-116 cells were significantly different at all time points with $p=0.03$ for day 3 and $p=0.001$ for days 4 and 5 .

The effect of $16-\mathrm{kDa}$ PRL on in vivo tumor growth was assessed using orthotopically implanted cells for the breast cancer cell lines or subcutaneously implanted cells for the breast and colon cancer cell lines. MDA-MB-231 and MCF-7 breast cancer cells and HCT-116 colon cancer cells were injected subcutaneously. At 8 weeks, all animals were sacrificed, and the tumors were immediately removed and measured to compare final size between tumors formed from EV- and 16-kDa-PRL-expressing cells. There was no difference in the size of the tumors arising from the breast cancer cells (Fig. 3) when comparing EV- or 16-kDa-PRLexpressing cells when using either Student's $t$ test or MannWhitney $U$ test. However, the HCT-116 cells produced dramatically different results. No tumors were present in the animals injected with HCT-116 cells expressing $16-\mathrm{kDa}$ PRL at 8 weeks postinjection, while all five animals injected with HCT-116 cells expressing empty vector developed tumors (Fig. 3).

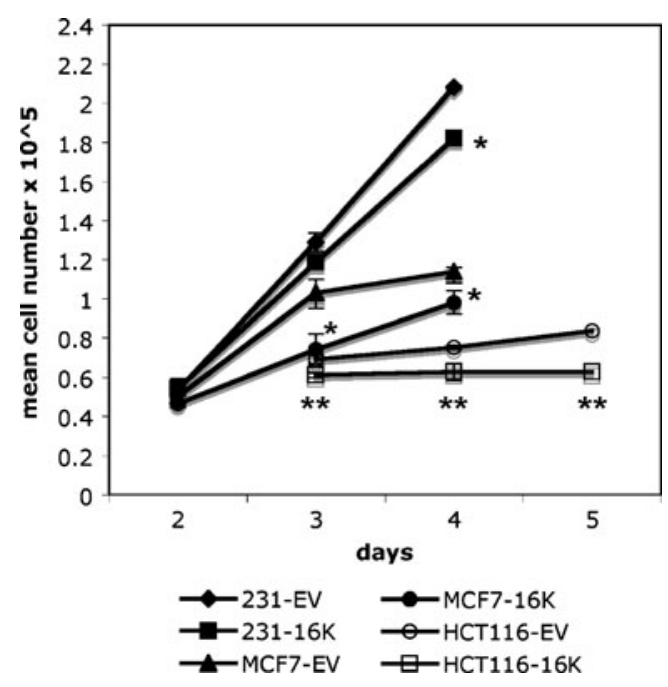

Fig. 2 The 16-kDa PRL expression reduces cell proliferation in vitro. MDA-MB-231, MCF-7, and HCT-116 cells expressing either 16-kDa PRL or empty vector was grown in media containing 5\% CSS. Each growth assay was repeated two (MCF-7, HCT-116) or three (MDAMB-231) times in triplicate. A representative example is shown here with standard deviations derived from the measures in triplicate. Error bars indicate SD with $* p<0.05$ and $* * p<0.01$. Filled diamonds $=$ MDA-MB-231/EV; filled squares $=$ MDA-MB-231/16K; filled triangles $=\mathrm{MCF}-7 / \mathrm{EV} ;$ filled circles $=\mathrm{MCF}-7 / 16 \mathrm{~K} ;$ empty circles $=$ HCT-116/EV; empty squares $=$ HCT-116/16K. 


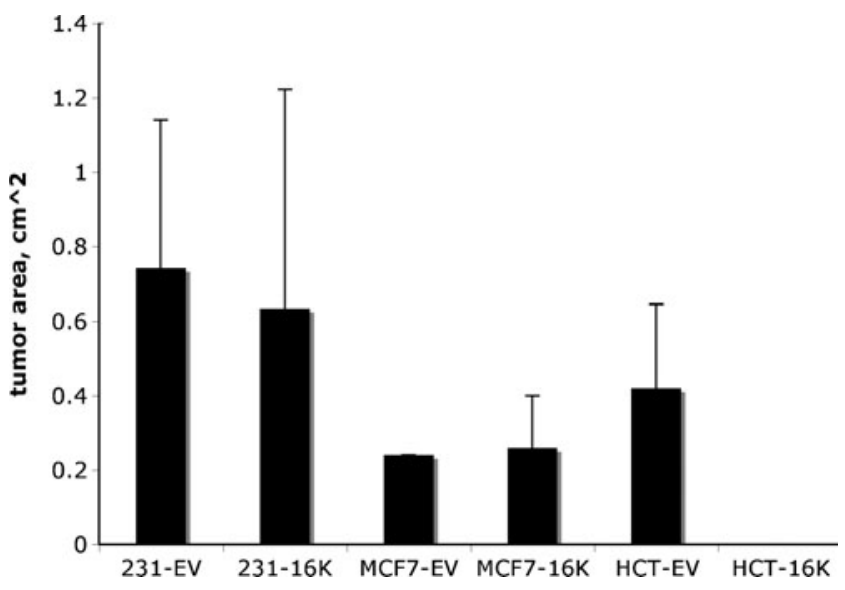

Fig. 3 The 16-kDa PRL expression has no effect on tumor size from breast cancer cells but inhibits tumor formation from colon cancer cells. Average tumor size $\left(\mathrm{cm}^{2}\right)$ at 8 -weeks postinjection (time of sacrifice) of MCF-7, MDA-MB-231, and HCT-116 cells expressing either 16-kDa PRL (16K) or empty vector (EV) injected subcutaneously. Tumor size did not differ significantly between EV and 16-kDa PRL expressing breast cancer cells; however, 16-kDa PRL expression inhibited tumor formation of HCT-116 cells. Error bars indicate SD, and asterisk indicates $p<0.05$ for $t$ test analysis.

In the orthotopic model, MDA-MB-231 and MCF-7 cells were injected into the abdominal mammary gland, and tumor growth was monitored using caliper measurements for 8 weeks. At 8 weeks, all animals were sacrificed, and the tumors were immediately removed and measured to compare final size between tumors formed from EV- and 16-kDa-PRL-expressing cells. Similar to the subcutaneous model, there was no difference in tumor size at sacrifice when comparing EV- to 16-kDa-PRL-expressing cells (data not shown), when using either Student's $t$ test or MannWhitney $U$ test. No statistical difference was seen when analyzing either tumor weight at sacrifice or number of tumors formed (data not shown). There also was no significant difference in cell proliferation index as measured by Ki-67 staining. Tumors from MDA-MB-231 cells expressing EV had on average $23.5 \%( \pm 16.8)$ of cells staining positive for Ki-67 while tumors from the MDAMB-231 expressing 16-kDa PRL had 13.4\% $( \pm 10.5)$ of cells positive. Similarly, tumors from MCF-7 cells expressing $\mathrm{EV}$ were $46.4 \%( \pm 2.3)$ positive, while tumors from MCF-7 cells expressing 16-kDa PRL were $49.9 \%( \pm 5.5)$ positive.

Previous research has demonstrated that $16-\mathrm{kDa}$ PRL is growth inhibitory to tumors derived from melanoma, prostate, and colon cancer cells lines, and this growth inhibition has been attributed to the antiangiogenic effects of 16-kDa PRL [14-16]. Since tumors arising from MDAMB-231 and MCF-7 breast cancer cell lines were not inhibited, additional experiments were performed to determine if the 16-kDa PRL ectopically expressed in these cells was antiangiogenic. In vivo angiogenic activity of MCF-7 and MDA-MB-231 cells expressing EV or $16-\mathrm{kDa}$ PRL was examined using a DIVAA [23]. For this assay, MCF-7, MDA-MB-231, and HCT-116 cells expressing EV or $16-\mathrm{kDa}$ PRL were placed in angioreactors that were implanted into the backs of athymic nu/nu mice for 9 days. For all three cell lines, there was little discernible recruitment of endothelial cells to the angioreactors containing cells expressing $16-\mathrm{kDa}$ PRL while angioreactors with EV cells demonstrated new blood vessel formation (Fig. 4a). Positive and negative controls were also included for each experiment. As expected, the positive control angioreactors containing FGF/VEGF recruited endothelial cells and new blood vessels were formed while there was no vascularization of the negative control angioreactors (Fig. 4a). When MCF-7 cells expressing 16$\mathrm{kDa}$ PRL were placed in the angioreactor along with FGF and VEGF, the vascularization seen with the positive control alone was inhibited (Fig. 4a). DIVAA results were quantified using densitometry analysis (Fig. 4b). Endothelial cells effectively migrated into the angioreactors of EVexpressing cells while there was minimal to no recruitment for all three cell types expressing 16-kDa PRL.

To further examine vascularization, MCF-7 cells expressing 16-kDa PRL and EV controls were injected orthotopically into nude mice. After tumors were palpable and reached a size of $0.25 \mathrm{~cm}^{2}$, high molecular weight FITC-dextran was injected into the tail vein of the mice to label functional blood vessels. Visualization of the FITCdextran in fixed tumors demonstrated that vascularization was greatly reduced in tumors expressing $16-\mathrm{kDa}$ PRL compared to EV (Fig. 5a). While there was a vast network of highly branched blood vessels throughout the tumors arising from the MCF-7-EV cells, only sparse, poorly branched blood vessels were seen on the tumors arising from the MCF-7-16-kDa PRL cells. These results were quantitated (Fig. 5b), demonstrating a significant reduction in functional blood vessels in MCF-7 cells expressing 16-kDa PRL.

\section{Discussion}

The effects of $16-\mathrm{kDa}$ PRL expression have been examined in prostate and colon cancer cell lines $[14,15]$ and more recently in melanoma models [16]. Prior results in prostate and colon cancer cell lines demonstrated that $16-\mathrm{kDa}$ PRL had no effect on cell proliferation in vitro but greatly reduced tumor formation and/or size in vivo $[14,15]$. Given the role of PRL in normal breast development and the growing literature suggesting that PRL levels are positively associated with human breast cancer [1], we sought to determine the effects of 16-kDa PRL expression in breast cancer cell lines. The MCF-7 and MDA-MB-231 cell lines 
Fig. 4 Directed in vivo angiogenesis assay (DIVAA). a Cells from either HCT-116, MDAMB-231, or MCF-7 empty vector $(E V)$ or $16-\mathrm{kDa}$ PRL $(16 K)$ transfected cells were placed into angioreactors as described in "Material and Methods." negative $=$ no added cells positive = addition of $\mathrm{FGF} /$

VEGF. $M C F-7-16 K+$ positive $=$ MCF-7 cells stably transfected with 16-kDa PRL placed in the angioreactor in the presence of FGF/VEGF. Photographs are representative from similar treatments from two separate experiments. The 16-kDa PRL expression inhibited blood vessel recruitment, even when mixed with FGF and VEGF. b Presence of blood vessels from DIVAA was quantified using NIH ImageJ from three separate angioreactors for each cell type and means compared using Mann-Whitney $U$ test. Error bars indicate SD and asterisk indicates $p=0.05$.
A.
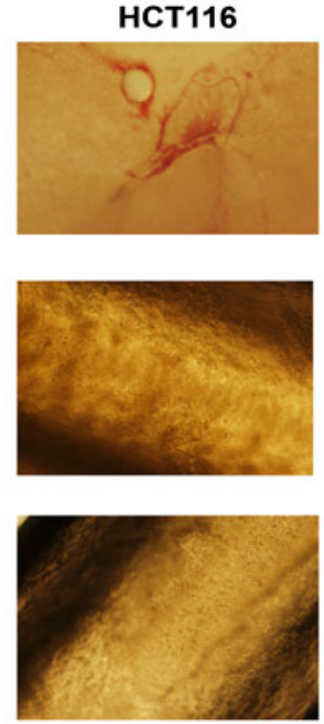

negative
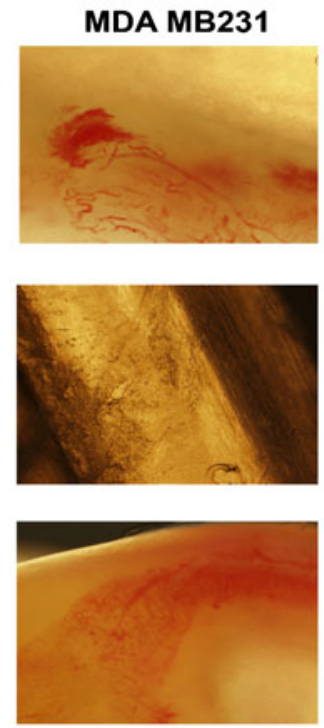

positive
MCF7
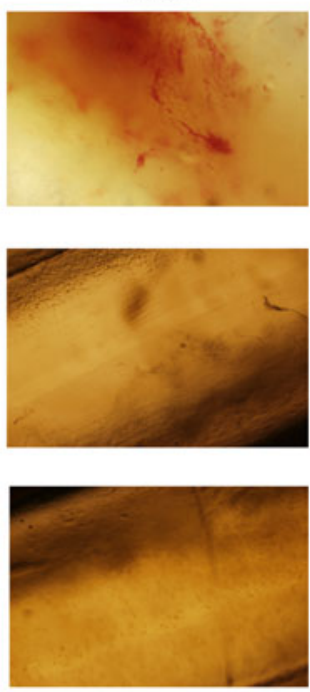

MCF7-16K + positive

B.

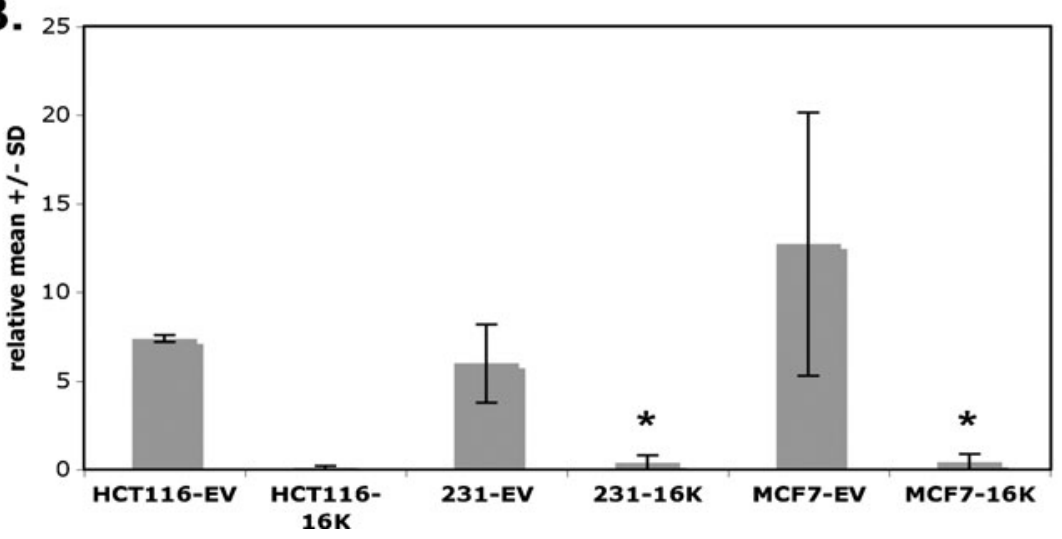

were chosen since these two cell lines vary greatly in ER and PRLR expression.

The 16-kDa PRL construct used here was developed based on results published by Piwnica et al., demonstrating that full-length 23-kDa PRL can be cleaved at three sites (aa 132, 147, and 150) by cathepsin D [9]. All three Nterminal PRL fragments are between 16 and $17 \mathrm{kDa}$ and are biologically active as demonstrated by inhibition of MAPK phosphorylation and proliferation of endothelial cells, as well as decreased vascularization in the chick allantoic membrane assay [9]. We chose to focus on the product cleaved from aa 150 as this approximated the $16-\mathrm{kDa}$ PRL found in samples of human pituitary adenomas [9]. Therefore, the $16-\mathrm{kDa}$ PRL isoform examined in this study was previously shown to result from cleavage by cathepsin $\mathrm{D}$, exhibit biological/antiangiogenic activity, and be present in human samples. In contrast, the 16-kDa PRL construct truncated at aa 123 in the studies focused on prostate cancer cell lines [15] and at aa 139 for expression in the colon [14] and melanoma cancer cell lines [16] are not generated by cathepsin $\mathrm{D}$ and have not yet been detected in vivo.

In spite of these differences in the $16-\mathrm{kDa}$ PRL expression constructs, our results in the HCT-116 cell lines are remarkably similar to those reported previously [14]. The HCT-116 cells reported here express 16-kDa PRL and also exhibit biological responses to $16-\mathrm{kDa}$ PRL expression. Specifically, we report that the HCT-116 cells expressing 16-kDa PRL demonstrate reduced cell proliferation in vitro, inhibition of angiogenesis by DIVAA, and reduced tumor formation.

The results from MCF-7 and MDA-MB-231 breast cancer cells differ greatly from the HCT-116 colon cancer cells in vivo. While expression of $16-\mathrm{kDa}$ PRL was antiangiogenic, as shown by both DIVAA and tail vein injection of FITC-dextran, there was no difference in tumor formation or tumor size in vivo for either breast cancer cell line. Tumor size is achieved through a balance of cell proliferation with apoptosis. Further experiments using 
A.

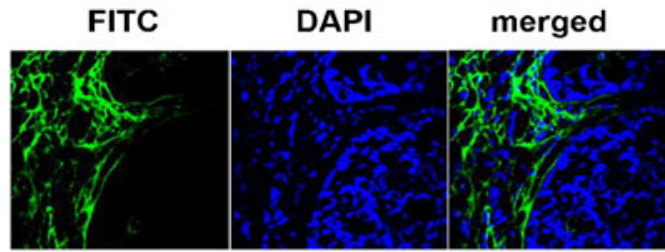

MCF7-EV
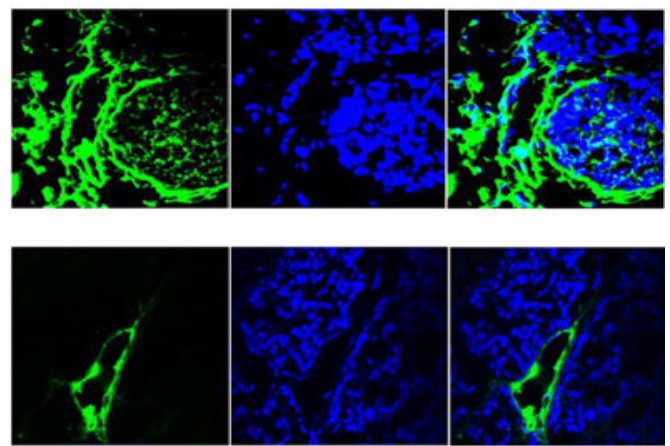

MCF-16K

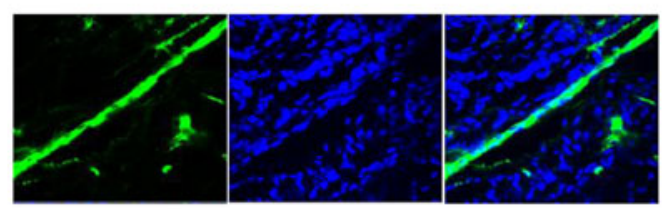

B.

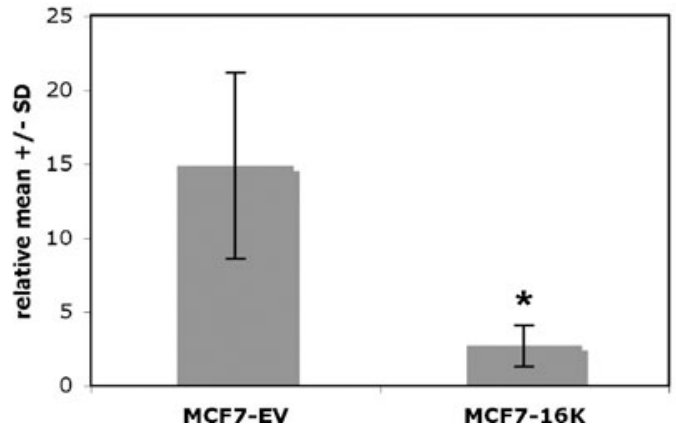

Fig. 5 FITC dextran visualization of vasculature. a Either MCF-7/EV or MCF-7/16K cells were injected into the mammary fat pads of nude mice. Once tumors reached $0.25-\mathrm{cm}^{2}$, FITC dextran was injected via the tail veins as described in "Material and Methods." MCF-7/16K cells exhibit reduced vascularization. Photographs are representative images from two separate tumors. b Presence of fluorescent-labeled vasculature was quantified using NIH ImageJ from separate images taken for each tumor type ( $n=7$ for MCF-7-EV, $n=6$ for MCF-7-16K). Error bars indicate SD and asterisk indicates $p=0.003$ for paired $t$ test analysis.

tumors from EV- and 16-kDa-PRL-expressing MDA-MB231 and MCF-7 cells demonstrated that there was no significant difference in Ki-67 staining between the two tumor types, suggesting that these two types of tumors exhibited similar cell proliferation. A previous publication demonstrated that 23-kDa PRL expression in MDA-MB435 cells resulted in increased tumor proliferation in vitro and tumor size in vivo [24]. In contrast, the 16-kDa PRL results presented here for both the MDA-MB-231 and MCF-7 breast cancer cell lines demonstrate a modest reduction in cell proliferation in vitro and no difference in tumor size in vivo. It is not entirely surprising that there was no difference in the in vivo environment. The breast cancer cell lines were injected orthotopically, thus permitting these cells contact with the normal epithelial and stromal cells in the mouse mammary gland and access to both local and systemic hormones and growth factors. The in vivo environment may contain additional factors that are more favorable to tumor formation and negate the antiangiogenesis and reduced proliferation effects seen with 16-kDa PRL expression. For example, the injected breast cancer cells would be exposed to endogenous hormones and growth factors in the mouse. Therefore, the properties of $16-\mathrm{kDa}$ PRL that would be inhibitory to tumor formation may be overwhelmed by endogenous protumorigenesis factors in the local and/or systemic environment.

In addition, an explanation for the difference in the in vivo results with respect to tumor development between the breast and colon cancer cell lines may be the exceptional sensitivity of breast cells to PRL as a mitogen. Unlike prostate and colon, complete breast development is dependent on PRL, where it is required for lobuloalveolar expansion and milk synthesis and secretion. Therefore, in both the PRLR-positive MCF-7 and PRLR-low MDA-MB231 cells, it is also possible that the mitogenic properties of $16-\mathrm{kDa}$ PRL may play a more dominant role in tumor formation than the antiangiogenic properties of this protein. The assays presented here clearly demonstrate that breast cancer cells expressing 16-kDa PRL could inhibit angiogenesis, yet this inhibition was not sufficient to reduce or slow tumor formation. The effects of $16-\mathrm{kDa}$ PRL on metastasis of the breast cancer cells were not explored in this study.

The antiangiogenic effects of $16-\mathrm{kDa}$ PRL have remained consistent over multiple different cancer cell lines (i.e., colon, prostate, melanoma, and now breast). It is possible that the antiangiogenic effects of $16-\mathrm{kDa}$ PRL are mediated through a receptor on endothelial cells that is distinct from PRLR [25]. Previous studies also have shown that the affinity of $16-\mathrm{kDa}$ PRL for the PRLR varies across different tissues [26]. While the affinity of 16-kDa PRL for receptors on mammary gland/breast cells has not been determined, it has been shown that $16-\mathrm{kDa}$ PRL can stimulate proliferation of these cells $[17,22]$. Therefore, in vivo, $16-\mathrm{kDa}$ PRL could be effectively inhibiting endothelial cell migration and/or proliferation while also still stimulating proliferation of breast cancer cells.

Our data also suggest that even cells low in PRLR expression, such as the MDA-MB-231 cells, may be responsive to the antiangiogenic effects of $16-\mathrm{kDa}$ PRL in vivo and demonstrate reduced cell proliferation in vitro. There may be several reasons for this response. The PRLR expressed on MDA-MB-231 may have increased sensitivity to $16-\mathrm{kDa}$ PRL binding, especially in the absence of 
competition with 23-kDa PRL. Alternatively, as discussed above, there may be a unique receptor for $16-\mathrm{kDa}$ PRL. At this time, we cannot state that the effects of $16-\mathrm{kDa}$ PRL are PRLR independent. Further validation of these results in additional breast cancer models is needed; however, the data presented herein suggest that the effects of $16-\mathrm{kDa}$ PRL may differ by cancer type. In breast cancer, it may be that the antiangiogenic effects of 16-kDa PRL on endothelial cells are balanced with the promitogenic effects this protein has on breast cells in vivo or that other in vivo factors can overcome the inhibition of tumor formation by $16-\mathrm{kDa}$ PRL expression. Further understanding the regulation of $16-\mathrm{kDa}$ PRL expression and activity may lead to additional insights into the PRL's role in human breast cancer, as well as illuminate additional treatment opportunities.

Acknowledgments This research was supported by the Center for Cancer Research, an Intramural Research Program of the National Cancer Institute. Dr. Faupel-Badger's research also was supported by the Cancer Prevention Fellowship Program, Center for Cancer Training, NCI.

\section{References}

1. Tworoger SS, Hankinson SE (2008) Prolactin and breast cancer etiology: an epidemiologic perspective. J Mammary Gland Biol Neoplasia 13:41-53

2. Clevenger CV, Furth PA, Hankinson SE, Schuler LA (2003) The role of prolactin in mammary carcinoma. Endocr Rev 24:1-27

3. Ginsburg E, Vonderhaar BK (1995) Prolactin synthesis and secretion by human breast cancer cells. Cancer Res 55:2591-2595

4. Fonseca ME, Ochoa R, Moran C, Zarate A (1991) Variations in the molecular forms of prolactin during the menstrual cycle, pregnancy and lactation. J Endocrinol Invest 14:907-912

5. Goldhar AS, Vonderhaar BK, Trott JF, Hovey RC (2005) Prolactin-induced expression of vascular endothelial growth factor via Egr-1. Mol Cell Endocrinol 232:9-19

6. Hovey RC, Goldhar AS, Baffi J, Vonderhaar BK (2001) Transcriptional regulation of vascular endothelial growth factor expression in epithelial and stromal cells during mouse mammary gland development. Mol Endocrinol 15:819-831

7. Clapp C, Gonzalez C, Macotela Y, Aranda J, Rivera JC, Garcia C et al (2006) Vasoinhibins: a family of N-terminal prolactin fragments that inhibit angiogenesis and vascular function. Front Horm Res 35:64-73

8. Clapp C (1987) Analysis of the proteolytic cleavage of prolactin by the mammary gland and liver of the rat: characterization of the cleaved and 16K forms. Endocrinology 121:2055-2064

9. Piwnica D, Touraine P, Struman I, Tabruyn S, Bolbach G, Clapp C et al (2004) Cathepsin D processes human prolactin into multiple 16K-like N-terminal fragments: study of their antiangiogenic properties and physiological relevance. Mol Endocrinol $18: 2522-2542$
10. Corbacho AM, Macotela Y, Nava G, Torner L, Duenas Z, Noris G et al (2000) Human umbilical vein endothelial cells express multiple prolactin isoforms. J Endocrinol 166:53-62

11. Duenas Z, Rivera JC, Quiroz-Mercado H, Aranda J, Macotela Y, Montes de Oca P et al (2004) Prolactin in eyes of patients with retinopathy of prematurity: implications for vascular regression. Invest Ophthalmol Vis Sci 45:2049-2055

12. Gonzalez C, Parra A, Ramirez-Peredo J, Garcia C, Rivera JC, Macotela $\mathrm{Y}$ et al (2007) Elevated vasoinhibins may contribute to endothelial cell dysfunction and low birth weight in preeclampsia. Lab Invest 87:1009-1017

13. Hilfiker-Kleiner D, Kaminski K, Podewski E, Bonda T, Schaefer A, Sliwa K et al (2007) A cathepsin D-cleaved $16 \mathrm{kDa}$ form of prolactin mediates postpartum cardiomyopathy. Cell 128:589-600

14. Bentzien F, Struman I, Martini JF, Martial J, Weiner R (2001) Expression of the antiangiogenic factor $16 \mathrm{~K}$ hPRL in human HCT116 colon cancer cells inhibits tumor growth in $\operatorname{Rag} 1(-/-)$ mice. Cancer Res 61:7356-7362

15. Kim J, Luo W, Chen DT, Earley K, Tunstead J, Yu-Lee LY et al (2003) Antitumor activity of the $16-\mathrm{kDa}$ prolactin fragment in prostate cancer. Cancer Res 63:386-393

16. Nguyen NQ, Cornet A, Blacher S, Tabruyn SP, Foidart JM, Noel $A$ et al (2007) Inhibition of tumor growth and metastasis establishment by adenovirus-mediated gene transfer delivery of the antiangiogenic factor 16K hPRL. Mol Ther 15:2094-2100

17. Clapp C, Martial JA, Guzman RC, Rentier-Delure F, Weiner RI (1993) The 16-kilodalton N-terminal fragment of human prolactin is a potent inhibitor of angiogenesis. Endocrinology 133:12921299

18. Trott JF, Hovey RC, Koduri S, Vonderhaar BK (2003) Alternative splicing to exon 11 of human prolactin receptor gene results in multiple isoforms including a secreted prolactin-binding protein. $\mathrm{J}$ Mol Endocrinol 30:31-47

19. Fields K, Kulig E, Lloyd RV (1993) Detection of prolactin messenger RNA in mammary and other normal and neoplastic tissues by polymerase chain reaction. Lab Invest 68:354-360

20. Fukumura D, Jain RK (2008) Imaging angiogenesis and the microenvironment. APMIS 116:695-715

21. Rowe RC, Cowden EA, Faiman C, Friesen HG (1983) Correlation of $\mathrm{Nb} 2$ bioassay and radioimmunoassay values for human serum prolactin. J Clin Endocrinol Metab 57:942-946

22. Clapp C, Sears PS, Russell DH, Richards J, Levay-Young BK, Nicoll CS (1988) Biological and immunological characterization of cleaved and $16 \mathrm{~K}$ forms of rat prolactin. Endocrinology 122:2892-2898

23. Guedez L, Rivera AM, Salloum R, Miller ML, Diegmueller JJ, Bungay PM et al (2003) Quantitative assessment of angiogenic responses by the directed in vivo angiogenesis assay. Am J Pathol 162:1431-1439

24. Liby K, Neltner B, Mohamet L, Menchen L, Ben-Jonathan N (2003) Prolactin overexpression by MDA-MB-435 human breast cancer cells accelerates tumor growth. Breast Cancer Res Treat 79:241-252

25. Clapp C, Weiner RI (1992) A specific, high affinity, saturable binding site for the 16-kilodalton fragment of prolactin on capillary endothelial cells. Endocrinology 130:1380-1386

26. Clapp C, Sears PS, Nicoll CS (1989) Binding studies with intact rat prolactin and a $16 \mathrm{~K}$ fragment of the hormone. Endocrinology 125:1054-1059 